\title{
Removing Even Crossings on Surfaces
}

\author{
Michael J. Pelsmajer ${ }^{1}$ \\ Department of Applied Mathematics \\ Illinois Institute of Technology \\ Chicago, Illinois 60616 \\ Marcus Schaefer ${ }^{2}$ \\ CTI, DePaul University \\ Chicago, Illinois 60604 \\ Daniel Stefankovič ${ }^{3}$ \\ Computer Science Department \\ University of Rochester \\ Rochester, NY 14627-0226
}

\begin{abstract}
We give a new, topological proof that the weak Hanani-Tutte theorem is true on orientable surfaces and extend the result to nonorientable surfaces. That is, we show that if a graph $G$ cannot be embedded on a surface $S$, then any drawing of $G$ on $S$ must contain two edges that cross an odd number of times. We apply the result and proof techniques to obtain new and old results about generalized thrackles, including that every bipartite generalized thrackle in a surface $S$ can be embedded in $S$. We also extend to arbitrary surfaces a result of Pach and Tóth that allows the redrawing of a graph so as to remove all crossings with even edges (an edge is even if it crosses every other edge an even number of times). From this result we can conclude that $\operatorname{cr}_{S}(G)$, the crossing number of a graph $G$ on surface $S$, is bounded by $2 \operatorname{ocr}_{S}(G)^{2}$, where $\operatorname{ocr}_{S}(G)$ is the odd crossing number of $G$ on surface $S$. Finally, we show that $\operatorname{ocr}_{S}(G)=\operatorname{cr}_{S}(G)$ whenever $\operatorname{ocr}_{S}(G) \leq 2$, for any surface $S$.
\end{abstract}

Keywords: Hanani-Tutte theorem, thrackles, odd crossing number, crossing number. 


\section{The weak Hanani-Tutte theorem on a surface}

The theorem of Hanani and Tutte states that every drawing in the plane of a nonplanar graph contains two non-adjacent edges which cross an odd number of times. ${ }^{4}$ There are several proofs of this theorem $[4,14,5,6,13,7]$ starting with the original papers by Hanani and Tutte. Kleitman's proof [6] is particularly short and elegant. All of these proofs invoke Kuratowski's theorem and then verify the result for subdivisions of $K_{3,3}$ and $K_{5}$. This approach seems hopeless for surfaces other than the plane (the list of excluded minors is not even known yet for the torus). ${ }^{5}$ In $[11,12]$ we gave a new proof of the Hanani-Tutte theorem in the plane which avoids Kuratowski's theorem and uses elementary topological methods only.

Cairns and Nikolayevsky showed that the weak Hanani-Tutte theorem is true for orientable surfaces with a short and elegant proof using homology theory [3, Lemma 3]. In the spirit of our earlier paper we give a new proof that is (1) algorithmic and (2) relies only on elementary/intuitive topological techniques, and (3) applies to nonorientable surfaces as well. Part of the argument is similar to our proof of the weak Hanani-Tutte theorem for the plane $[11,12]$ which proceeds by contracting edges. New ideas are needed to deal with the case where every edge is a loop.

We call an edge even if it crosses every other edge an even number of times.

Theorem 1.1 If $G$ can be drawn on a surface $S$ so that all its edges are even, then $G$ can be embedded on that surface, i.e. drawn crossing-free, without changing the embedding scheme.

\section{Generalized Thrackles}

A graph is a thrackle if it can be drawn such that any pair of edges intersects exactly once, where a common endpoint of two edges counts as an intersection of these two edges. Generalized thrackles were introduced by Woodall in 1972 [1, p. 359-363]. A generalized thrackle is a graph that can be drawn

$\overline{1 \text { Email: }}$ pelsmajer@iit.edu

2 Email: mschaefer@cti.depaul.edu

3 Email: stefanko@cs.rochester.edu

4 We make the usual assumptions on drawings of graphs, see [8, page 230].

5 For the purposes of this abstract, a surface is a compact, connected surface without boundary. By the classification theorem for surfaces, an orientable surface is homeomorphic to a sphere with a number of handles attached, and a nonorientable surface is homeomorphic to a sphere with a number of crosscaps. 
such that any pair of edges intersects an odd number of times (again counting endpoints).

Cairns and Nikolayevsky proved the weak Hanani-Tutte theorem for orientable surfaces in order to apply it to generalized thrackles, thereby extending earlier work of Lovász, Pach, and Szegedy and establishing tighter bounds on the number of edges of thrackles and generalized thrackles [3].

Theorem 2.1 (Cairns, Nikolayevsky) Let $G$ be bipartite. Then $G$ is a generalized thrackle on some orientable surface if and only if $G$ can be embedded on that surface.

Our topological approach handles generalized thrackles very naturally and we simplify and improve several results on generalized thrackles. Note that the distinction between crossings and intersections in the definition of generalized thrackles is not essential. Two edges cross (rather than intersect) if they intersect at a point which is not an endpoint of either edge (we also assume they do not touch). It is easy to see that a graph is a generalized thrackle if and only if it can be drawn such that any pair of edges crosses (rather than intersects) an odd number of times.

The following lemma establishes a link between generalized thrackles and the weak Hanani-Tutte theorem. It cannot be extended to all graphs as it is well-known that there are planar graphs that are not generalized thrackles (e.g. the wheel $W_{4}$, see [3]).

Lemma 2.2 If $D$ is a drawing of a bipartite graph $G$ on some surface $S$, then we can find a drawing $D^{\prime}$ of $G$ on $S$ such that two edges e and $f$ cross oddly in $D$ if and only if they cross evenly in $D^{\prime}$. In other words, we can flip the crossing parity of all pairs of edges.

The lemma allows an easy topological proof of the following result which extends Theorem 2.1 to nonorientable surfaces.

Theorem 2.3 Let $G$ be bipartite. Then $G$ is a generalized thrackle on some surface if and only if $G$ can be embedded on that surface.

In a subsequent paper, Cairns and Nikoleyevsky study generalized thrackles of non-bipartite graphs on orientable surfaces, using the following definition. A parity embedding of a graph is a drawing without crossings such that even cycles are two-sided curves and odd cycles are one-sided curves. They prove [2, Theorem 2]: 
Theorem 2.4 (Cairns, Nikolayevsky) $G$ is a generalized thrackle on an orientable surface $S$ if and only if $G$ has a parity embedding on the (nonorientable) surface obtained by adding a crosscap to $S$.

Again we can give a very simple proof of this result, which originally had quite a lengthy proof. Our methods work just as well when $S$ is nonorientable, once we see how to extend the definition of parity embedding appropriately. Let $S^{\prime}$ be the surface obtained by adding the crosscap $X$ to the orientable surface $S$. Observe that a closed curve in $S^{\prime}$ is one-sided if and only if it passes through $X$ an odd number of times. Therefore a parity embedding in $S^{\prime}$ is an embedding in which the parity of cycle length equals the parity of the number of times it passes through $X$. Now, for any nonorientable surface with a specified crosscap $X$, we define an $X$-parity embedding to be an embedding in which a cycle is odd if and only if it passes through $X$ an odd number of times. (For an orientable surface with one added crosscap $X$, parity embedding is identical to $X$-parity embedding.) We can now state our result, which generalizes Theorem 2.4.

Theorem 2.5 $G$ is a generalized thrackle on a surface $S$ if and only if $G$ has an $X$-parity embedding on the surface obtained by adding a crosscap $X$ to $S$, with the same embedding scheme.

\section{Removing even crossings in arbitrary surfaces}

The previous sections might leave the impression that results related to the Hanani-Tutte theorem easily generalize to arbitrary surfaces, but this is not actually the case as we will now see. In the plane, the weak Hanani-Tutte theorem can be strengthened as follows:

Theorem 3.1 (Pach, Tóth) If $D$ is a drawing of $G$ in the plane, and $E_{0}$ is the set of even edges in $D$, then $G$ can be drawn in the plane so that no edge in $E_{0}$ is involved in any crossings.

Pach and Tóth applied their result to establish a relationship between two different notions of crossing numbers. The crossing number, $\operatorname{cr}(G)$, of a graph $G$ is the smallest number of crossings in a drawing of $G$. The odd crossing number, ocr $(G)$, is the smallest number of pairs of edges that cross oddly in a drawing of $G$. By definition $\operatorname{ocr}(G) \leq \operatorname{cr}(G)$; however there are graphs for which the two numbers differ [10]. On the other hand, Pach and Tóth showed that $\operatorname{cr}(G) \leq 2 \operatorname{ocr}(G)^{2}$.

The redrawing procedure used in the proof of Theorem 3.1 can lead to an 
increase of the odd crossing number, and will, therefore, probably not lead to better bounds of $\operatorname{cr}(G)$ in terms of $\operatorname{ocr}(G)$ (a linear bound is suspected). In a previous paper we showed that Theorem 3.1 can be strengthened to avoid an increase in the odd crossing number:

Theorem 3.2 (Pelsmajer, Schaefer, Štefankovič) If $D$ is a drawing of $G$ in the plane, and $E_{0}$ is the set of even edges in $D$, then $G$ can be drawn in the plane so that no edge in $E_{0}$ is involved in any crossings and there are no new pairs of edges that cross an odd number of times.

If we change the point of view from the plane to other surfaces, the situation changes: we can construct examples that show that the stronger Theorem 3.2 fails on any surface other than the sphere. The original result of Pach and Tóth, however, is true for arbitrary surfaces, as we can show.

Theorem 3.3 If $D$ is a drawing of a connected graph $G$ in some surface $S$, and $E_{0}$ is the set of even edges in $D$, then $G$ can be drawn in $S$ so that no edge in $E_{0}$ is involved in any crossings.

While the odd crossing number of the drawing does get increased in the proof of this theorem, the result is strong enough to extend the result by Pach and Tóth that $\operatorname{cr}(G) \leq 2 \operatorname{ocr}(G)^{2}$ in the plane to any surface, using essentially the same proof given by Pach and Tóth for the planar case [9].

Corollary 3.4 For any surface $S$ we have

$$
\operatorname{cr}_{S}(G) \leq 2 \operatorname{ocr}_{S}(G)^{2}
$$

Using Theorem 3.2 we were able to show that for the plane, $\operatorname{ocr}(G)=\operatorname{cr}(G)$ whenever $\operatorname{ocr}(G) \leq 3$. Even though Theorem 3.1 cannot be strengthened to an analogue of Theorem 3.2, it does allow us to derive the following result.

Theorem 3.5 If $G$ is a graph and $S$ is a surface with $\operatorname{ocr}_{S}(G) \leq 2$, then $\operatorname{ocr}(G)=\operatorname{cr}(G)$.

\section{References}

[1] Unsolved problems. In Combinatorics (Proc. Conf. Combinatorial Math., Math. Inst., Oxford, 1972), pages 351-363. Inst. Math. Appl., Southend, 1972. Chairman: P. Erdős. 
[2] G. Cairns and Y. Nikolayevsky. Generalized thrackles drawings of non-bipartite graphs. Preprint, http://www.latrobe.edu.au/mathstats/staff/cairns/ dvi/generalized.pdf.

[3] G. Cairns and Y. Nikolayevsky. Bounds for generalized thrackles. Discrete Comput. Geom., 23(2):191-206, 2000.

[4] C. C. H. Hanani). Über wesentlich unplättbare Kurven im drei-dimensionalen Raume. Fundamenta Mathematicae, 23:135-142, 1934.

[5] H. Harborth. Parity of numbers of crossings for complete $n$-partite graphs. Math. Slovaca, 26(2):77-95, 1976.

[6] D. J. Kleitman. A note on the parity of the number of crossings of a graph. $J$. Combinatorial Theory Ser. B, 21(1):88-89, 1976.

[7] S. Norine. Pfaffian graphs, t-joins, and crossing numbers. To be published by Combinatorica.

[8] J. Pach. Geometric graph theory. In Handbook of discrete and computational geometry, 2nd edition, CRC Press Ser. Discrete Math. Appl., pages 219-238. CRC, Boca Raton, FL, 2004.

[9] J. Pach and G. Tóth. Which crossing number is it anyway? J. Combin. Theory Ser. B, 80(2):225-246, 2000.

[10] M. J. Pelsmajer, M. Schaefer, and D. Stefankovič. Odd crossing number is not crossing number. In P. Healy and N. S. Nikolov, editors, Graph Drawing, volume 3843 of Lecture Notes in Computer Science, pages 386-396. Springer, 2005 .

[11] M. J. Pelsmajer, M. Schaefer, and D. Štefankovič. Removing even crossings. In S. Felsner, editor, 2005 European Conference on Combinatorics, Graph Theory and Applications (EuroComb '05), volume AE of DMTCS Proceedings, pages 105-109. Discrete Mathematics and Theoretical Computer Science, April 2005.

[12] M. J. Pelsmajer, M. Schaefer, and D. Štefankovič. Removing even crossings. J. Combin. Theory Ser. B, 2006. To appear.

[13] L. A. Székely. A successful concept for measuring non-planarity of graphs: the crossing number. Discrete Math., 276(1-3):331-352, 2004. 6th International Conference on Graph Theory.

[14] W. T. Tutte. Toward a theory of crossing numbers. J. Combinatorial Theory, 8:45-53, 1970. 